\title{
Time-dependent couplings and crossover length scales in nonequilibrium surface roughening
}

\author{
Marc Pradas, ${ }^{1}$ Juan M. López, ${ }^{2, *}$ and A. Hernández-Machado ${ }^{1}$ \\ ${ }^{1}$ Departament d'Estructura i Constituents de la Matèria, Universitat de Barcelona, Avinguda Diagonal 647, E-08028 Barcelona, Spain \\ ${ }^{2}$ Instituto de Física de Cantabria (IFCA), CSIC-UC, E-39005 Santander, Spain
}

(Received 12 March 2007; revised manuscript received 18 April 2007; published 11 July 2007)

\begin{abstract}
We show that time-dependent couplings may lead to nontrivial scaling properties of the surface fluctuations of the asymptotic regime in nonequilibrium kinetic roughening models. Three typical situations are studied. In the case of a crossover between two different rough regimes, the time-dependent coupling may result in anomalous scaling for scales above the crossover length. In a different setting, for a crossover from a rough to either a flat or damping regime, the time-dependent crossover length may conspire to produce a rough surface, although the most relevant term tends to flatten the surface. In addition, our analysis sheds light into an existing debate in the problem of spontaneous imbibition, where time-dependent couplings naturally arise in theoretical models and experiments.
\end{abstract}

DOI: 10.1103/PhysRevE.76.010102

The theory of surface growth has applications to phenomena that cover a wide range of length scales from nanometers to millimeters, including the growth of thin films from an incoming flux of atoms [1-3], fluid imbibition in porous media [4], and propagation of fracture cracks [5], among many others. It is often observed that surfaces kinetically roughen and become scale invariant.

In kinetic roughening details of the interactions are largely irrelevant in the mathematical description of the critical properties of the surface at long wavelengths, akin to critical point phenomena. Therefore, in the hydrodynamic limit, one can describe surface growth in $d+1$ dimensions by the stochastic equation

$$
\frac{\partial h}{\partial t}=\mathcal{G}(\nabla h)+\eta(\mathbf{x}, t)
$$

where $h(\mathbf{x}, t)$ is the height of the interface at substrate position $\mathbf{x}$ and time $t$. The functional $\mathcal{G}(\nabla h)$ depends on the specific model and should satisfy all the symmetries and conservation laws. The external noise $\eta(\mathbf{x}, t)$ describes the random driving forces acting on the surface, for instance, the influx of particles in a deposition processes.

We shall be discussing here surface growth models with local coupling among degrees of freedom, i.e., growth equations that include only terms that depend on derivatives of the height, $Y=\nabla h$. In the spirit of Ginzburg-Landau-Wilson theory of critical phenomena, the functional $\mathcal{G}(Y)$ is constructed as the leading-order expansion in powers of the argument $Y$, its derivatives, and combinations thereof. One explicitly avoids including terms that are incompatible with the symmetries of the problem. The corresponding expansion takes the form of a sum, $\mathcal{G}(\Upsilon)=\Sigma_{\mathrm{I}} \omega_{\mathrm{I}} \Phi_{\mathrm{I}}(\mathbf{x}, t)$, where $\omega_{\mathrm{I}}$ are the coupling constants and $\Phi_{\mathrm{I}}(\mathbf{x}, t)$ are the local operators in the terminology of the renormalization group (RG). Local operators correspond to the combinations of degrees of freedom and its derivatives, which typically include surface diffusion $\nabla^{2} h$, curvature diffusion $\nabla^{4} h$, and in general higher-

\footnotetext{
*lopez@ifca.unican.es
}

order diffusion terms $\nabla^{2 m} h$. Also nonlinear terms usually appear, $(\nabla h)^{2 n}, \nabla^{2 n}(\nabla h)^{2 m}$, and so on. The asymptotic long wavelength limit is governed by the most relevant terms in the RG sense. Higher-order terms are nonetheless important in describing crossover effects before the truly asymptotic behavior is reached. This leads to the existence of crossover length scales and characteristic times at which one can observe the true asymptotic scaling behavior of the system. In the case of time-independent coupling parameters the analysis of crossover length scales is relatively simple and very well studied [1-3]. However, little is known about less common situations in which the couplings depend explicitly on time. Examples include the problem of a stable phase growing at the expense of a metastable phase [6], and spontaneous imbibition [4], among others. Indeed, the problem of spontaneous imbibition has been a subject of great interest in the last few years [4,7-11]. Different theoretical approaches have arrived at the conclusion that, for small deviations around of the mean position $H(t)$, the fluid-fluid interface (in Fourier space) is given by

$$
\frac{\partial \hat{h}_{k}(t)}{\partial t}=-\sigma K|k| k^{2} \hat{h}_{k}-\dot{H}(t)|k| \hat{h}_{k}+K|k| \hat{\eta}_{k},
$$

where $\sigma$ and $K$ are the surface tension and permeability constants, respectively, and the average position follows Washburn's law $H(t)=\sqrt{H_{0}^{2}+2 a t}$ and arises from mass conservation. There is an interesting debate regarding the role of this time-dependent coupling in the scaling observed in both simulations [7,10,11] and experiments [12-14]. This problem has largely motivated our study of the scaling of the surface fluctuations in systems where couplings depend explicitly on time.

In this Rapid Communication we study the interplay between a crossover length scale growing in time and the dynamic correlation length characterizing the kinetic roughening process. We find nontrivial scaling properties, including anomalous roughening $[15,16]$. The long time limit scaling behavior strongly depends on the nature of the phases that the dynamic crossover separates. We focus on three typical cases that cover the most important situations one can find: 
(i) Crossover between two different rough regimes, (ii) crossover from a rough to a flat regime, and (iii) the existence of a damping term, where scale-invariant fluctuations are damped over a certain length scale that varies with time. We exemplify our general scaling analysis with simple model systems in which dimensional analysis gives the exact exponents. In order to gain analytical understanding we restrict ourselves to linear model examples that allow exact computation of the critical exponents. Results are compared with numerical simulations.

Crossover between two different rough regimes. Consider a growing surface described by Eq. (1). Invariance under translation along the growth and substrate directions as well as invariance in the election of the time origin rule out an explicit dependence of $\mathcal{G}$ on $h, \mathbf{x}$, and $t$. These symmetry requirements alone lead to scale invariant growth in a generic fashion [1]. Let us consider the two most relevant terms in the leading-order expansion of $\mathcal{G}(\nabla h)$ in the hydrodynamic limit, so that we have $\mathcal{G}=\omega_{\mathrm{I}} \Phi_{\mathrm{I}}(x, t)+\omega_{\mathrm{II}} \Phi_{\mathrm{II}}(x, t)+$ higherorder terms. Let the $\Phi_{\mathrm{I}}$ term be more relevant (in the RG sense) than $\Phi_{\mathrm{II}}$. We first discuss the case in which Eq. (1) exhibits crossover between two different rough regimes. This means $\omega_{\mathrm{II}} \Phi_{\mathrm{II}}(x, t)$ is relevant at short scales, while $\omega_{\mathrm{I}} \Phi_{\mathrm{I}}(x, t)$ becomes the most dominant in the long wavelength limit. Hence one expects to find an early times (short scales) regime with scaling exponents $\alpha^{(\mathrm{II})}$ and $z^{(\mathrm{II})}$ that crosses over to the true asymptotic regime, governed by the operator $\Phi_{\mathrm{I}}$, with exponents $\alpha^{(\mathrm{I})}$ and $z^{(\mathrm{I})}$. Dimensional analysis indicates that there exists a crossover length $\ell_{\times} \sim\left(\omega_{\mathrm{II}} / \omega_{\mathrm{I}}\right)^{1 / q}$ for some exponent $q$. This is the typical length above which the most relevant term $\omega_{\mathrm{I}} \Phi_{\mathrm{I}}$ takes over. This is likely the most common situation of crossover behavior in surface growth.

The question we wish to address here is how this picture is modified when the most relevant operator's coupling, $\omega_{\mathrm{I}}$, decreases in time. The most interesting situation naturally arises for a power-law decay of the coupling $\omega_{\mathrm{I}}(t) \sim t^{-\gamma}$, so that the term $\omega_{\mathrm{I}}(t) \Phi_{\mathrm{I}}$ becomes effectively less relevant for longer times as compared with the $\omega_{\text {II }} \Phi_{\text {II }}$ term. In this case the crossover length diverges in time as a power-law, $\ell_{\times}(t) \sim t^{\gamma / q}$, and the interplay with the other relevant scale in the problem, namely, correlation length $\xi(t) \sim t^{1 / z}$, will give rise to an interesting behavior. On scales smaller than $\ell_{\times}(t)$ the less relevant term II dominates, while we expect a crossover to the asymptotic regime I for $\xi(t) \gg \ell_{\times}(t)$. The caveat is now that the observable scaling regimes crucially depend on the exponent of the coupling $\gamma$. If $\gamma<q / z^{(\mathrm{II})}$, the crossover does take place as described above. On the contrary, for $\gamma>q / z^{(\mathrm{II})}$, the crossover length grows at an exponentially faster rate than the correlation length. Therefore, in this case the time-dependent coupling effectively takes the crossover length to scales much larger than those that can be correlated by the dynamics at any finite time. As a consequence the system will never cross over to regime I, and only the "less" relevant operator II governs the scaling regime observable for arbitrarily large system sizes.

We now study a simple $1+1$ dimensional model that exemplifies the crossover behavior between two different scaling regimes discussed above. We consider Eq. (1) with $\Phi_{\mathrm{I}}=\nabla^{2} h$ and $\Phi_{\mathrm{II}}=-\nabla^{4} h$, describing diffusive coupling. Then we have the growth equation

$$
\frac{\partial h}{\partial t}=\nu(t) \nabla^{2} h-\nabla^{4} h+\eta(x, t),
$$

where we have rescaled all the couplings but $\nu(t)$ to unity. The noise is $\delta$ correlated, $\left\langle\eta(x, t) \eta\left(x^{\prime}, t^{\prime}\right)\right\rangle$ $=2 \delta\left(x-x^{\prime}\right) \delta\left(t-t^{\prime}\right)$. Higher-order diffusion terms, $\nabla^{2 n} h$, might be present, but are irrelevant for the scaling behavior. Actually, even the $\nabla^{4} h$ term is irrelevant as compared with $\nabla^{2} h$ if the couplings are independent of time. Consider now a decaying coupling $\nu(t) \sim t^{-\gamma}[17]$. Balancing the two gradient terms in Eq. (3) one finds that they become comparable at the typical crossover scale $\ell_{\times}(t) \sim t^{\gamma / 2}$, with the exponent $q=2$ in our previous analysis.

Dimensional analysis tells us that the kinetic roughening process is governed by the fourth derivative term (that we label as II) on scales smaller than $\ell_{x}$, while the diffusion term should dominate on much larger scales (that we label as regime I). Below the crossover, for $\xi(t) \ll \ell_{\times}(t)$, Eq. (3) has a correlation length $\xi(t) \sim t^{1 / z^{(I I)}}$, where $z^{(\mathrm{II})}=4$, given by the $\nabla^{4} h$ term dynamics. Crossover to the asymptotic regime I takes place when the correlation length reaches the crossover length $t^{1 / z^{(\mathrm{II})}} \sim t^{\gamma / 2}$. Following our previous analysis we expect that regime I can only be reached if $\gamma<2 / z^{(\mathrm{II})}$, i.e., $\gamma<1 / 2$. On the contrary, for $\gamma>1 / 2$ the crossover is wiped out and the less relevant operator II (in RG sense) does govern the scaling in the long wavelengths limit as well. This analysis is in excellent agreement with simulations as shown in Fig. 1.

Let us now study the scaling properties in the case $\gamma<1 / 2$, where one expects two different regimes with different critical exponents. Smaller scales, regime II, are simply governed by the dynamics of the curvature diffusion term $\nabla^{4} h$ and we find the well-known results $z^{(\mathrm{II})}=4, \alpha^{(\mathrm{II})}=3 / 2$, and the local roughness exponent $\alpha_{\text {loc }}^{(\mathrm{II})}=1[15,16]$. Above the crossover, $\xi(t) \gg \ell_{\times}$, the system is expected to be in regime I, and a scaling analysis gives the exact global exponents $\alpha^{(\mathrm{I})}=(1+\gamma) /[2(1-\gamma)], z^{(\mathrm{I})}=2 /(1-\gamma), \beta^{(\mathrm{I})}=(1+\gamma) / 4$, and the scaling relation $\alpha^{(\mathrm{I})}=z^{(\mathrm{I})} \beta^{(\mathrm{I})}$ is fulfilled. Figure 1 shows that this scaling analysis is in excellent agreement with a numerical integration of the model (3).

However, even for $\gamma<1 / 2$ the asymptotic scaling behavior is nontrivial, since the time-dependent coupling $\nu(t)$ leads to anomalous scaling of the local surface fluctuations. This is more clearly proved in Fourier space in terms of the structure factor (or spectral power spectrum), $S(k, t)$ $=\langle\hat{h}(k, t) \hat{h}(-k, t)\rangle$, where $\hat{h}(k, t)$ is the Fourier transform of the surface in a system of lateral size $L, \hat{h}(k, t)$ $=L^{-1 / 2} \sum_{x}[h(k, t)-\bar{h}(t)] \exp (i k x)$. From the growth equation (3) and neglecting the $\nabla^{4} h$ term we obtain

$$
S(k, t) \sim k^{-2} t^{\gamma} s\left(k^{2} t^{1-\gamma}\right),
$$

where the scaling function $s(u)$ has the asymptotes $s(u) \sim u$ for $u \ll 1$ and $s(u) \sim$ const for $u \gg 1$ [18]. The temporal shift in the power spectrum $t^{\gamma}$ implies anomalous scaling of the local fluctuations. The theory of anomalous scaling $[15,16]$ tells us that the scaling relation $\gamma=2\left(\alpha^{(\mathrm{I})}-\alpha_{\text {loc }}^{(\mathrm{I})}\right) / z^{(\mathrm{I})}$ must be fulfilled. We then find that, according to the spectral density 

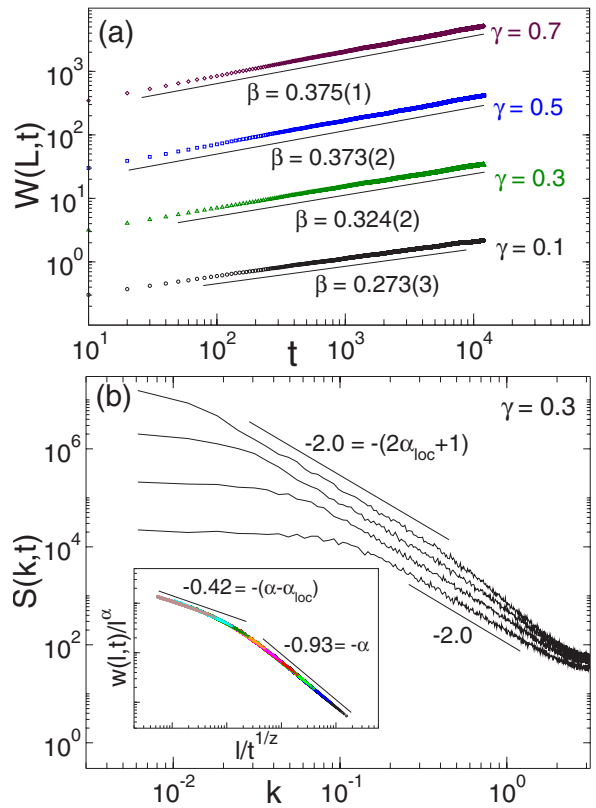

FIG. 1. (Color online) Crossover from rough to rough. Numerical integration of Eq. (3) using a time step $\Delta t=10^{-2}$ and $c=10^{-3}$ in a system size $L=1024$. All results were averaged over 200 realizations. (a) Global width of the interface, computed for different $\gamma$ values. The theoretical prediction for the growth exponent $\beta^{(\mathrm{I})}=(1+\gamma) / 4$ for $\gamma<1 / 2$ fully agrees with simulations. For any $\gamma>1 / 2$ we obtain $\beta=3 / 8$, as expected. The curves are vertically shifted for clarity. (b) Power spectrum for $\gamma=0.3$ evaluated at times $10,10^{2}, 10^{3}$, and $10^{4}$. Anomalous scaling with a local roughness exponent $\alpha_{\text {loc }}^{(\mathrm{I})}=0.5$ is observed. The inset shows a data collapse of the local width data for $\alpha^{(\mathrm{I})}=0.93(1)$ and $z^{(\mathrm{I})}=2.86(4)$ to be compared with the predicted values $\alpha^{(\mathrm{I})}=0.928$ and $z^{(\mathrm{I})}=2.857$ for $\gamma=0.3$.

(4), the scaling in the asymptotic regime (regime I) is anomalous in this case with a local roughness exponent $\alpha_{\mathrm{loc}}^{(\mathrm{I})}=1 / 2$. Note that the local roughness exponent does not depend on the coupling exponent $\gamma$ and self-affinity $\alpha^{(\mathrm{I})}=\alpha_{\text {loc }}^{(\mathrm{I})}=1 / 2$ is recovered whenever the coupling is time independent $(\gamma=0)$. A comparison with numerical simulations for $\gamma=0.3$ is shown in Fig. 1.

Crossover from a rough to a flat regime. Another interesting situation occurs when the most relevant operator happens to lead to a flat surface. In this case the crossover is expected to take place from an early times (short scales) rough regime II, with roughness exponent $\alpha^{(\mathrm{II})}>0$ to an asymptotic flat regime, where $\alpha^{(\mathrm{I})}=0$. Again, a time-dependent crossover length leads to nontrivial scaling properties in this case. Below the crossover we expect a rough phase dominated by the $\Phi_{\text {II }}$ term with exponents $\alpha^{(\mathrm{II})}>0$ and $z^{(\mathrm{II})}$. Above the crossover, however, surface correlations cannot evolve any longer since the operator $\Phi_{\mathrm{I}}$ does not amplify surface fluctuations (note that $\alpha^{(\mathrm{I})}=0$ and $z^{(\mathrm{I})}=0$ ). This leads to an asymptotic roughness exponent identical to that in the early regime. Moreover, it is worth it to stress here that the dynamical length scale $\sim t^{1 / z^{(\mathrm{I})}}$ becomes a constant above the crossover (note that $z^{(\mathrm{I})}=0$ ). Therefore, above the crossover the only relevant scale left in the problem scales as $\sim t^{\gamma / q}$. This must be identified with the correlation length $\xi(t)$ above the cross-

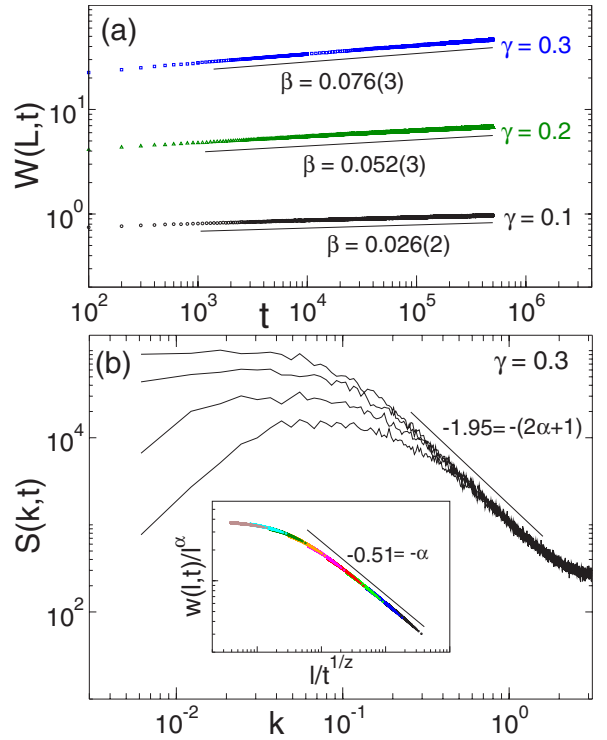

FIG. 2. (Color online) Crossover from rough to flat. Numerical integration of Eq. (3) with conserved noise in a system size $L=1024$. Results were averaged over 200 realizations. (a) Global width for different $\gamma$. The growth exponent was found to fit with the theoretical value $\beta=\gamma / 4$. The curves are vertically shifted for clarity. (b) Power spectrum for $\gamma=0.3$ at times $10^{3}, 10^{4}, 10^{5}$, and $5 \times 10^{5}$. It shows a roughness exponent $\alpha=0.48$ (3). The inset shows a data collapse of the local width data using $\alpha=0.51(2)$ and $z=6.63(4)$, which agrees with the scaling prediction $z=2 / \gamma$ for $\gamma=0.3$.

over. We therefore conclude that the time-dependent coupling leads to an asymptotic regime with exponents $\alpha=\alpha^{\text {(II) }}$ and $z=q / \gamma$, instead of those that would naively arise from the operator I, $\alpha^{(\mathrm{I})}=z^{(\mathrm{I})}=0$. It is remarkable that, although the most relevant term tends to flatten the surface, the timedependent coupling conspires to produce a rough surface. The roughness exponent is inherited from the early time (short scale) phase. Meanwhile, the time-dependent crossover length becomes the only relevant scale above the crossover, which is to be associated with the dynamical exponent $z$ of the system in the long times regime.

To exemplify this general scaling analysis we now study a simple $1+1$ dimensional model system exhibiting a crossover between rough regime on short scales to a flat surface regime in the long wavelengths limit. Let us consider the same growth model as in Eq. (3), but the noise is now conserved, $\left\langle\eta_{c}(x, t) \eta_{c}\left(x^{\prime}, t^{\prime}\right)\right\rangle=-2 \nabla^{2} \delta\left(x-x^{\prime}\right) \delta\left(t-t^{\prime}\right)$. Note that the surface diffusion operator $\nabla^{2} h$ leads to flat surface fluctuations $\alpha^{(\mathrm{I})}=z^{(\mathrm{I})}=0$ in the presence of conserved noise.

On small scales the surface is expected to be dominated by the less relevant $\nabla^{4} h$ term, so we obtain $z^{(\mathrm{II})}=4$ and $\alpha^{(\mathrm{II})}=\alpha_{\mathrm{loc}}^{(\mathrm{II})}=1 / 2$ in the early times (short scales) regime. Above the crossover the $\nabla^{2} h$ term governs the surface fluctuations and we find $z^{(\mathrm{I})}=2 /(1-\gamma)$ and $\alpha^{(\mathrm{I})}$ $=-(1-3 \gamma) /[2(1-\gamma)]$ by simple power counting. Therefore, for values $0<\gamma \leqslant 1 / 3$ we have the desired situation of a crossover from a rough to a flat regime. According to our scaling argument we expect scaling in the asymptotic regime with a roughness exponent $\alpha=\alpha^{(\mathrm{II})}=1 / 2$ and a dynamical 
exponent $z=2 / \gamma$, instead of the trivial values $\alpha^{(\mathrm{I})}=0$ and $z^{(\mathrm{I})}=0$ that would naively correspond to the $\nabla^{2} h$ operator with conserved noise. A comparison with a numerical integration of the model for a time-dependent coupling with exponents $0<\gamma<1 / 3$ shows an excellent agreement with this analysis (see Fig. 2).

Crossover to a damped regime. Finally, we briefly discuss the case in which surface fluctuations become damped over a certain scale. This means that, for small deviations around the mean surface height, the terms $-h,-h^{2}, \ldots$ are to be included in the growth equation. These terms break the $h \rightarrow h+c$ symmetry and therefore also break scale invariance. Let us consider a growth model with the leading-order expansion $\mathcal{G}=-\omega(t) h(x, t)+\omega_{\mathrm{II}} \Phi_{\mathrm{II}}(x, t)+$ higher-order terms. In such a way the operator $\Phi_{\mathrm{II}}$ is less relevant than the $-h$ damping term, but it would lead to scale-invariant behavior in the absence of damping. Following a scaling analysis similar to that of the previous cases we find that for $z^{\text {(II) }}<q / \gamma$ the crossover to the damping regime will never take place. So, in all respects, the damping term is irrelevant and the surface will exhibit the scaling behavior corresponding to the operator II. On the contrary, if $z^{(\mathrm{II})}>q / \gamma$, the crossover occurs when $\xi(t) \sim \ell_{\times}(t)$. The asymptotic regime in this case does exhibit scaling but with the nontrivial exponents $\alpha=\alpha^{(\mathrm{II})}$ and $z=q / \gamma$, for the same reasons as in the previously discussed rough-to-flat case. Simulation results of the simple model (not shown), $\partial_{t} h=-\omega(t) h+\nabla^{2} h+\eta$, are also in excellent agreement with this scaling analysis.

We have shown that the presence of time-dependent coupling in nonequilibrium surface roughening has important implications in the scaling properties of the asymptotic regime. We have focused on local models that include the two most relevant terms separated by a crossover length scale growing in time, $\ell_{\times}(t) \sim t^{\gamma / q}$, which conspires with the correlation length of the system $\xi(t) \sim t^{1 / z}$ to give highly nontrivial scaling properties. In the case of a crossover between two rough regimes, the surface may exhibit anomalous roughening, directly related to the value of $\gamma \neq 0$. On the other hand, in the case of a crossover from a rough to either a flat or damping regime, the dynamical crossover length $\ell_{\times}(t) \sim t^{\gamma / q}$ is the only relevant scale above the crossover and this immediately leads to an asymptotic rough regime with a dynamic exponent $z=q / \gamma$. A rough regime appears despite the most relevant term that tends to flatten the surface. Remarkably, this is precisely the numerical result found in recent studies in spontaneous imbibition $[4,7,10,11]$, where the interface growth is described by Eq. (2). Our results show that these numerical results can be understood in the wider context of kinetic roughening in systems with timedependent couplings.

This work is supported by the DGI of the Ministerio de Educación y Ciencia (Spain) through Grants No. FIS200612253-C06-04 and No. FIS2006-12253-C06-05.
[1] A.-L. Barabási and H. E. Stanley, Fractal Concepts in Surface Growth (Cambridge University Press, Cambridge, 1995).

[2] J. Krug, Adv. Phys. 46, 139 (1997).

[3] A. Pimpinelli and J. Villain, Physics of Crystal Growth (Cambridge University Press, Cambridge, 1998).

[4] M. Alava, M. Dubé, and M. Rost, Adv. Phys. 53, 83 (2004).

[5] E. Bouchaud, J. Phys.: Condens. Matter 9, 4319 (1997).

[6] E. Hernandez-García, T. Ala-Nissila, and M. Grant, Europhys. Lett. 21, 401 (1993).

[7] M. Dubé et al., Phys. Rev. Lett. 83, 1628 (1999); M. Dubé, M. Rost, and M. Alava, Eur. Phys. J. B 15, 691 (2000); M. Dubé et al., ibid. 15, 701 (2000).

[8] A. Hernández-Machado et al., Europhys. Lett. 55, 194 (2001).

[9] E. Pauné and J. Casademunt, Phys. Rev. Lett. 90, 144504 (2003).

[10] M. Pradas and A. Hernández-Machado, Phys. Rev. E 74, 041608 (2006).

[11] T. Laurila et al., Eur. Phys. J. B 46, 553 (2005); T. Laurila, C. Tong, S. Majaniemi, and T. Ala-Nissila, Phys. Rev. E 74, 041601 (2006).

[12] J. Soriano, J. J. Ramasco, M. A. Rodriguez, A. Hernandez-
Machado, and J. Ortin, Phys. Rev. Lett. 89, 026102 (2002); J. Soriano, J. Ortín, and A. Hernández-Machado, Phys. Rev. E 66, 031603 (2002).

[13] J. Soriano et al., Phys. Rev. Lett. 95, 104501 (2005).

[14] D. Geromichalos, F. Mugele, and S. Herminghaus, Phys. Rev. Lett. 89, 104503 (2002).

[15] J. M. López, M. A. Rodríguez, and R. Cuerno, Phys. Rev. E 56, 3993 (1997); Physica A 246, 329 (1997).

[16] J. M. López, Phys. Rev. Lett. 83, 4594 (1999); J. J. Ramasco, J. M. López, and M. A. Rodríguez, ibid. 84, 2199 (2000); J. M. López, M. Castro, and R. Gallego, ibid. 94, 166103 (2005).

[17] An arbitrary small constant $c$ is required to avoid the singularity at $t=0$, so that $\nu \sim \nu_{0}(c+t)^{-\gamma}$. This has no significance for our analysis but it must be taken into account in numerical simulations.

[18] Note that Eq. (4) describes the surface scaling before saturation. For finite systems the steady state occurs at very long times, $t \sim L^{2 / \gamma}$, where one recovers $S(k) \sim k^{-4}$. We are interested here in the intermediate regime before saturation is reached, in which both operators $k^{-2}$ and $k^{-4}$ play a role at different scales. 